\title{
On-orbit Noise Characterization for MODIS Reflective Solar Bands
}

\author{
X. Xiong \\ Sciences and Exploration Directorate, NASA/GSFC, Greenbelt, MD 20771 \\ Xiaoxiong.Xiong-1@nasa.gov \\ 301-614-5957 \\ $X$. Xie and A. Angal \\ Science Systems and Applications, Inc., 10210 Greenbelt Road, Lanham, MD 20706
}

\begin{abstract}
Since launch, the Moderate Resolution Imaging Spectroradiometer (MODIS) has operated successfully on-board the NASA Earth Observing System (EOS) Terra spacecraft for more than eight years, and on-board the EOS Aqua spacecraft for nearly 6 years. MODIS is a passive crosstrack scanning radiometer that makes observations in 36 spectral bands with spectral wavelengths from visible (VIS) to long-wave infrared (LWIR) and has spatial resolutions at nadir of $0.25 \mathrm{~km}$ (bands 1-2), $0.5 \mathrm{~km}$ (bands 3-7), and $1 \mathrm{~km}$ (bands 8-36). MODIS bands $1-19$ and 26 are the reflective solar bands (RSB) with wavelengths from 0.41 to $2.2 \mu \mathrm{m}$. They are calibrated on-orbit using an on-board solar diffuser (SD) and a solar diffuser stability monitor (SDSM) system. The remaining 16 spectral bands are the thermal emissive bands (TEB) with wavelengths above 3.5 $\mu \mathrm{m}$. For MODIS RSB, the Level 1B (L1B) calibration algorithm produces top of the atmosphere (TOA) reflectance factors and radiances for every pixel of the Earth view. The sensor radiometric calibration accuracy, specified at each spectral band's typical scene radiance, is $2 \%$ for the RSB reflectance factors and 5\% for the RSB radiances. Also specified at the typical scene radiance is the detector signal-to-noise ratio (SNR), a key sensor performance parameter that directly impacts its radiometric calibration accuracy and stability, as well as the image quality. For both Terra and Aqua MODIS, the RSB detector SNR was fully characterized pre-launch using a large aperture spherical integration source (SIS) operated at different lamp configurations (radiance levels). Multiple data samples and scans were collected at each lamp configuration.
\end{abstract}

In this paper, we describe an on-orbit SNR characterization approach developed to evaluate and track MODIS RSB detector performance. This approach uses RSB detector responses to the SD when it is illuminated by sunlight during its on-orbit calibration. Each $1 \mathrm{~km}$ resolution detector collects 50 data samples each scan over the SD sector. Because of spacecraft movement, the solar illumination angles on the SD panel continually change. Consequently, each detector's response to the SD, even within the same scan, vary continuously with the source illumination onto the SD. Typical scan-by-scan variation of each detector's response is much higher than the sample-tosample variation within the same scan. In order to perform on-orbit SNR characterization, MODIS RSB detector responses to the solar illumination reflected from the SD panel must be corrected for factors due to variations of the solar angles and the SD bi-directional reflectance factor (BRF). This approach enables RSB SNR characterization to be performed at different response levels for each detector. It has been recently applied by the MODIS Characterization 
Support Team (MCST) at NASA/GSFC to update the RSB detector SNR for both Terra and Aqua MODIS over their entire missions. On-orbit results show that both Terra and Aqua MODIS RSB detectors have performed well since launch. Except for a few noisy or inoperable detectors which were identified pre-launch, most RSB detectors continue to meet the SNR design requirements and are able to maintain satisfactory short-term stability. A comparison of on-orbit noise characterization results with results derived from pre-launch calibration and characterization is also provided in this paper. 